\title{
L. On determinant notation
}

\section{E. J. Nanson M.A.}

To cite this article: E. J. Nanson M.A. (1897) L. On determinant notation, Philosophical Magazine Series 5, 44:270, 396-400, DOI: 10.1080/14786449708621081

To link to this article: http://dx.doi.org/10.1080/14786449708621081

$$
\text { 曲 Published online: } 08 \text { May } 2009 .
$$

Submit your article to this journal 전

Џll Article views: 2

Q View related articles ¿ 
but agrees very well with the results obtained by Volkmann, Hall, Sentis, Rayleigh, and others.

In conclusion I wish to thank Professor Rowland and Dr. Ames for their suggestions and encouragement throughout the entire course of this work.

Johns Hopkins University, June 1897.

L. On Determinant Notation. By E. J. Nanson, M.A.*

THE notation for a determinant introduced in 1861 by

H. J. S. Smith has been almost universally adopted on account of its extreme simplicity and clearness. That notation is that the determinant

is denoted by

$$
\left|\begin{array}{cccc}
a_{11} & a_{12} & \ldots & a_{1 n} \\
a_{21} & a_{22} & \ldots & a_{2 n} \\
\vdots & \vdots & \vdots \\
a_{n_{1}} & a_{n 2} & \ldots & a_{n n}
\end{array}\right|
$$

$$
\left|a_{p q}\right| \quad(p, q=1, \ldots n ! \text {. }
$$

Now just as it is convenient to have this simple notation for the determinant, so also is it convenient to have a simple notation for the array of symbols used to form the determinant, or, more generally, for an array in which the number of rows is not necessarily the same as the number of columns.

It is therefore proposed to use the notation

for the array

$$
\left(a_{p q}\right) \quad(p=1, \ldots m ; q=1, \ldots n) ;
$$

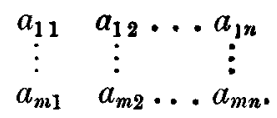

This notation is particularly convenient in dealing with determinants which can be divided by horizontal and vertical lines into blocks, such that all elements in the same block follow the same law. For instance, the bordered determinant

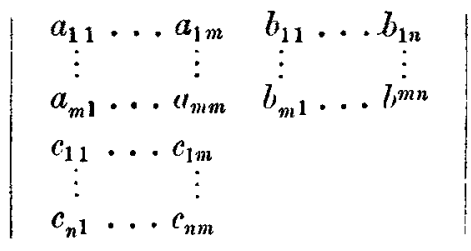

* Communicated by the Author. 
may be denoted by

$$
\left.\left|\begin{array}{ll}
\left(a_{p q}\right) & \left(b_{p s}\right) \\
\left(c_{r q}\right) &
\end{array}\right| \begin{array}{l}
p, q=1, \ldots m \\
r, s=1, \ldots n
\end{array}\right) .
$$

As an example take the theorem given long ago by Sylvester*, as an illustration of the power of this umbral notation.

In the notation now suggested that theorem may be expressed as follows :-

If

$$
e_{r s}=\left|\begin{array}{cc}
\left(a_{p q}\right) & \left(b_{p s}\right) \\
\left(\dot{c_{r q}}\right) & d_{r s}
\end{array}\right| \quad(p, q=1, \ldots m) ;
$$

then

$$
\left|e_{r s}\right|=\left|a_{p q}\right|^{n-1} \cdot\left|\begin{array}{ll}
\left(a_{p q}\right) & \left(b_{p s}\right) \\
\left(c_{r q}\right) & \left(d_{r s}\right)
\end{array}\right|(r, s=1, \ldots n) .
$$

Proofs of this theorem have been given by Scott $\dagger$, Frobenius $\ddagger$, and Netto $\S$. But in no one of these proofs is the fundamental simplicity of the theorem made evident.

In the first place, by the general law of extension due to Muir I, the theorem is at once seen to be true, because it is merely the extensional of the obvious identity

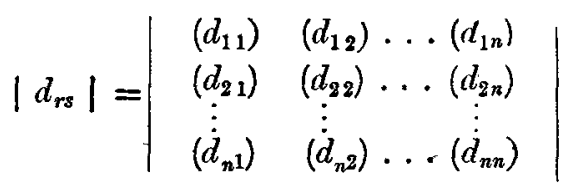

In the second place, the theorem may be proved directly by the following elementary method.

Multiply columns 1 to $m$ of the determinant $\Delta$, where

$$
\Delta=\left|\begin{array}{ll}
\left(a_{p q}\right) & \left(b_{p s}\right) \\
\left(c_{r q}\right) & \left(d_{r s}\right)
\end{array}\right|\left(\begin{array}{c}
p, q=1, \ldots m \\
r, s=1, \ldots n
\end{array}\right),
$$

by $\lambda_{s,}, \ldots \lambda_{s m}$, add to column $m+s$, and do this for all values of $s$. Then, provided the multipliers $\lambda$ are taken so that

$$
\Sigma_{q} a_{p q} \lambda_{s q}+h_{p s}=0, \text {. . . . . }
$$

* Phil, Mag. April 1851, p. 297.

+ Proc. Lond. Math. Soc. vol. xiv. p. 92.

$\ddagger$ Crelle, vol. cxiv. p. 189.

$\$$ Acta Math. vol. xvii. p. 202.

II Trans. R. S. E. xxx. p. 1; 'Theory of Determinants,' p. 213. 
we get

where $f_{r s}$ is given by

$$
\begin{aligned}
& \Delta=\left|\begin{array}{cc}
\left(a_{p q}\right) & \\
\left(c_{r q}\right) & \left(f_{r s}\right)
\end{array}\right| \\
& =\left|a_{p q}\right| \cdot\left|f_{r s}\right|, \ldots . .
\end{aligned}
$$

$$
\Sigma_{q} c_{r q} \lambda_{s q}+d_{r s}=f_{r s^{*}} \cdot \text {. . . . }
$$

Now, eliminating $\lambda$ from (1), (3), we get

$$
\left|\begin{array}{cc}
\left(a_{p q}\right) & \left(h_{p s}\right) \\
\left(c_{r q}\right) & d_{r s}-f_{r s}
\end{array}\right|=0
$$

whence $f_{r s}$ is given by

$$
\begin{aligned}
\left|c_{p q}\right| f_{r s} & =\left|\begin{array}{cc}
\left(a_{p q}\right) & \left(b_{p s}\right) \\
\left(c_{r q}\right) & d_{r s}
\end{array}\right| \\
& =e_{r s}
\end{aligned}
$$

by definition of $e_{r s}$. Therefore, by (2)

$$
\begin{aligned}
\left|a_{p q}\right|^{n-1} \Delta & =\left|a_{p q}\right|^{n} \cdot\left|f_{r s}\right| \\
& =\left|e_{r s}\right|,
\end{aligned}
$$

which is the theorem to be proved.

Sylvester speaks of this theorem as "one of the most prolific in results of any with which I am acquainted," and further says: "It is obvious that without the aid of my system of umbral or biliteral notation this important theorem could not be made the subject of statement without an enormous periphrasis, and could never have been made the object of distinct contemplation or proof."

The theorem just proved is a particular case of another theorem given by Sylvester in the same paper. Before giving a statement of this second theorem it is necessary to explain a notation for a minor determinant and also for what may be called a minor array.

If $\theta, \phi$ be $\nu$-ads from $1, \ldots n$. then the minor formed from the $\nu$ rows $\theta$ and the $\nu$ columns $\phi$ of the determinant

$$
\left|a_{p q}\right| \quad(p, g=1, \ldots n)
$$

may be denoted by $\left|a_{\theta \phi}\right|$.

In like manner, if $\theta$ be a $\mu$-ad from $1, \ldots m$ and $\phi$ a $\nu$-ad from $1, \ldots n$, then the array formed by the elements common 
to the $\mu$ rows $\theta$ and the $\nu$ columns $\phi$ of the array

$$
\left(a_{p q}\right) \quad(p=1, \ldots m ; q=1, \ldots n)
$$

may be denoted by $\left(a_{\theta \phi}\right)$. In particular the array formed by all the elements in the $\mu$ rows $\theta$ may be denoted by $\left(a_{\theta q}\right)$.

With this notation Sylvester's second theorem may be stated as follows:-

If $\theta, \phi$ be $y$-ads from $1, \ldots n$ and

then

$$
\mathrm{E}_{\theta \Phi}=\left|\begin{array}{cc}
\left(a_{p q}\right) & \left(b_{p \varphi}\right) \\
\left(c_{\theta q}\right) & \left(d_{\theta \phi}\right)
\end{array}\right|(p, q=1, \ldots m),
$$

where

$$
\left|\mathrm{E}_{\theta \phi}\right|=\left|a_{p q}\right|^{\lambda} \cdot\left|\begin{array}{ll}
\left(a_{p q}\right) & \left(b_{p s}\right) \\
\left(c_{r q}\right) & \left(d_{r s}\right)
\end{array}\right|^{\lambda^{\prime}}(r, s=1, \ldots n),
$$

$$
\begin{aligned}
\lambda & =\frac{(n-1)(n-2) \ldots(n-\nu+1)}{1.2 \ldots v}, \\
\lambda^{\prime} & =\frac{(n-1)(n-2) \ldots(n-v)}{1.2 \ldots(v-1)} .
\end{aligned}
$$

Proofs of this theorem have been given by Reiss*, Picquet $\dagger$, Scott $\ddagger$, and Van Velzer $\S$. Scott deduces the second theorem from the first in the following simple way :-

By the first theorem we have

$$
\mathrm{A}^{\nu-1} \mathrm{E}_{\theta \phi}=\left|e_{\theta \phi}\right|,
$$

where $\mathrm{A}=\left|a_{p q}\right|$. Hence the determinant

$$
\left|\mathrm{A}^{v-1} \mathrm{E}_{\theta \phi}\right|,
$$

which is of order $\lambda+\lambda^{\prime}$, is the $\nu$-th compound of $\left|e_{r s}\right|$ and is therefore, by Franke's theorem, equal to $\left|e_{r s}\right|{ }^{\lambda}$. But by the first theorem we have $\left|e_{r s}\right|=\mathrm{A}^{n-1} \Delta$, and hence we readily find $\left|\mathrm{E}_{\theta \phi}\right|=\mathrm{A}^{\lambda} \Delta^{\lambda^{\prime}}$.

Van Velzer remarks that this proof " seems to leave nothing to be desired, either in simplicity or rigour." Sylvester's second theorem is, however, merely the extensional of Franke's theorem already quoted. It is certainly remarkable that a very general theorem enunciated without proof by Sylvester

* Beiträge zur Th. der. Det. p. 34 (1867).

$\dagger$ Journal de l'Ecole Pol. Cah. xlv. p. 216.

$\ddagger$ I'roc. Lond, Math. Soc. xiv. p. 93.

$\S$ An. Journ. Math. vi. p. 168. 
in 1851 contains as its simplest special case the theorem of Franke, which was not discovered until 1862 .

As another example in illustration of the block notation take the determinant identities associated with the name of Schweins. These are found, after Cayley*, by expanding the determinant

$$
\left|\begin{array}{ll}
\left(a_{p q}\right) & \left(b_{p s}\right) \\
\left(c_{r q}\right) &
\end{array}\right|
$$

where $p=1, \ldots m ; q=1, \ldots n ; r=1, \ldots n-k ; s=1, \ldots n-k$, in two different ways and equating the results. This process gives, by Laplace's theorem,

$$
\Sigma_{\theta}(-1)^{\lambda}\left|\begin{array}{c}
\left(a_{\theta q}\right) \\
\left(c_{r q}\right)
\end{array}\right| \cdot\left|b_{\theta^{\prime} s}\right|=\Sigma_{\phi}(-1)^{\mu}\left|\left(a_{p \Phi}\right)\left(b_{p s}\right)\right| \cdot\left|c_{r \phi^{\prime}}\right|,
$$

where $\theta, \phi$ are $k$-ads from $1, \ldots m ; 1, \ldots n$ respectively; $\theta^{\prime}, \phi^{\prime}$ are the sets complimentary to $\theta, \phi$ respectively; and $\lambda, \mu$ are the sums of the numbers in the sets $\theta, \phi$ respectively.

The general extensional of this identity is

$$
\begin{aligned}
& \Sigma_{\theta}(-1)^{\lambda}\left|\begin{array}{ll}
\left(a_{\theta q}\right) & \left(d_{\theta u}\right) \\
\left(c_{r q}\right) & \left(d_{r u}\right) \\
\left(e_{t q}\right) & \left(f_{t u}\right)
\end{array}\right| \cdot\left|\begin{array}{ll}
\left(b_{\theta^{\prime} s}\right) & \left(d_{\theta^{\prime}}\right) \\
\left(e_{t s}\right) & \left(f_{t u}\right)
\end{array}\right| \\
& =\Sigma_{\varphi}(-1)^{\mu}\left|\begin{array}{lll}
\left(a_{p \phi}\right) & \left(b_{p s}\right) & \left(d_{p u}\right) \\
\left(e_{t \phi}\right) & \left(e_{t s}\right) & \left(f_{t u}\right)
\end{array}\right| \cdot\left|\begin{array}{ll}
\left(c_{r \phi^{\prime}}\right) & \left(d_{r u}\right) \\
\left(e_{t \phi^{\prime}}\right) & \left(f_{t u}\right)
\end{array}\right|,
\end{aligned}
$$

where $t, u=1, \ldots z$.

Muir + tells us that "the notation which in our time would almost certainly be chosen for the statement of such identities" as those of Schweins, "is the umbral notation of Sylvester."

The object of this short note is to show that the umbral notation is not always the most convenient, and that in some cases at all events the block notation conduces to brevity and lucidity.

Melbourne, July 31, 1897.

* C. M. P. 9 and 676.

$\dagger$ Phil. Mag. Nov. 1884, p. 422. 